Article

\title{
Increasing the Level of IRS-1 and Insulin Pathway Sensitivity by Natural Product Carainterol A
}

\author{
Kaiqing Ma ${ }^{1}$, Yanhong Miao ${ }^{1,2}$, Yao Gao ${ }^{1}$, Junsheng Tian ${ }^{1}$, Li Gao ${ }^{1}$, Deyong Ye ${ }^{3, *}$ \\ and Xuemei Qin ${ }^{1, *}$ \\ 1 Modern Research Center for Traditional Chinese Medicine, Shanxi University, Taiyuan 030006, China; \\ makaiqing@sxu.edu.cn(K.M.); myj15530149989@163.com (Y.M.); 18734823114@163.com (Y.G.); \\ jstian@sxu.edu.cn (J.T.); gaoli87@sxu.edu.cn (L.G.) \\ 2 College of Chemistry and Chemical Engineering, Shanxi University, Taiyuan 030006, China \\ 3 Department of Medicinal Chemistry, School of Pharmacy, Fudan University, Shanghai 201203, China \\ * Correspondence: dyye@shmu.edu.cn (D.Y.); qinxm@sxu.edu.cn (X.Q.); \\ Tel.: +86-215-198-0117 (D.Y.); +86-351-701-1501 (X.Q.)
}

Academic Editor: Derek J. McPhee

Received: 1 September 2016; Accepted: 23 September 2016; Published: 29 September 2016

\begin{abstract}
Carainterol A is a eudesmane sesquiterpenoid extracted from Caragana intermedia. We have reported that carainterol A showed potent glucose consumption activity in $\mathrm{C}_{2} \mathrm{C}_{12}$ muscle cells and the $\mathrm{db} / \mathrm{db}$ mouse model. However, the mechanism of the hypoglycemic effect of carainterol $\mathrm{A}$ remains elusive. In this article, we present a network pharmacology approach to predict the target and signaling pathway of carainterol A which was subsequently validated in HepG2 cells. It was demonstrated that carainterol A could increase the protein levels of IRS-1 and the downstream protein kinase AKT phosphorylation at a low micromolar level. These findings suggest that carainterol A can be a valuable lead compound and a promising chemical probe for the insulin signaling pathway.
\end{abstract}

Keywords: carainterol A; network pharmacology; insulin receptor substrate 1; insulin signaling pathway

\section{Introduction}

Natural products and their derivatives have been an invaluable source for drug discovery [1]. Sesquiterpenoids have aroused considerable interest and attracted continuous attention as they embody impressive architectural diversity, stereochemical intricacies and pronounced biological activities. These properties continue to render sesquiterpenoids as exciting objectives for organic chemists and biologists [2]. Carainterol A (1), an architecturally complex and unique eudesmane sesquiterpenoid natural product (Figure 1), was isolated from the aerial part of Caragana intermedia which is a frequently used herb in traditional Chinese medicine. In our previous study, it is demonstrated that carainterol A showed potent activity in increasing glucose consumption in $\mathrm{C}_{2} \mathrm{C}_{12}$ muscle cells. Furthermore, it also displayed glucose consumption in a $\mathrm{db} / \mathrm{db}$ mouse model with a MIC (minimum inhibitory concentration) value that is equivalent to that of metformin [3]. Due to its unique structure and significant biological activity, we have completed the total synthesis of carainterol A [4]. However, the pharmacological targets associated the mechanism of carainterol A remained elusive.

Identifying functional targets and clarifying the molecular mechanism of action of bioactive natural products have been proven to be particularly challenging [5]. Network pharmacology, a system biology-based methodology, has been recently employed to provide an effective mapping of the yet unexplored target space of natural products and a systematic way to extend the druggable space of proteins implicated in different complex diseases [6]. This approach has been utilized successfully for the study of the molecular mechanism of traditional Chinese medicine (TCM) [7] and single 
multi-targeted natural products [6,8]. For example, the PharmMapper server was used as a valuable tool for identifying targets for several natural products such as sinapic acid [9]. The mode of action by the network analysis (MANTRA) method was developed to discover the proapoptotic effect of pinosylvin [10]. Therefore, network pharmacology is supposed to be a promising approach for learning the mechanism of carainterol A.

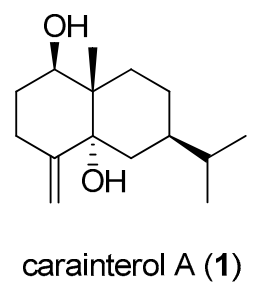

Figure 1. Structure of carainterol A.

In this study, carainterol A was analyzed using the PharmMapper and ClueGO to predict the target proteins and related pathways, respectively. Furthermore, carainterol A was evaluated in the HepG2 cells in order to clarify its biochemical action. We demonstrated that carainterol A could increase insulin pathway sensitivity through increasing the protein levels of IRS-1 and the downstream protein kinase AKT phosphorylation at a low micromolar level.

\section{Results}

\subsection{Target and Pathway Predictions}

After the removal of duplication and standardization, 118 potential molecular targets were retrieved based on the fitting score from high to low. These potential molecular targets are involved in a variety of physiological and/or pathological processes. Among the 118 protein targets, 46 targets (39\%) including IGF1R, INSR, MDM2, PPARG, RXRA, PTN1, RXRB and RARG have been reported to be related to diabetes by previous studies. Sixteen pairs of compound-target interactions were found and their fitting scores of docking were greater than 3.5 (Table 1). For example, protein tyrosine phosphatase 1B (PTN1), the enzyme that catalyzes tyrosine dephosphorylation, functions as anegative regulator in both insulin and leptin signaling [11]. The mutations resulting in the loss of function of peroxisome proliferator-activated receptor $\gamma(\operatorname{PPAR} \gamma)$ underlie the development of severe insulin resistance and overt type 2 diabetes [12]. Retinoid X receptor (RXR) agonists function as insulin sensitizers in obese mice [13]. Therefore, carainterol A may interact with these proteins and transcription factor to show potent activity in increasing glucose consumption. This is consistent with our previous findings on the pharmacological effects of carainterol A in diabetes which further argued that the PharmMapper approach was feasible and robust in the target identification of natural products.

Table 1. The fitting score of docking of carainterol A with potential targets.

\begin{tabular}{cccccc}
\hline No. & Target Gene & Uniprot ID & Protein Name & Fit & Norm Fit \\
\hline 1 & GSTA1 & P08263 & Glutathione S-transferase A1 & 4.341 & 0.5426 \\
2 & RXRA & P19793 & Retinoic acid receptor RXR- $\alpha$ & 3.916 & 0.5594 \\
3 & SULT2A1 & Q06520 & Bile salt sulfotransferase & 3.77 & 0.7541 \\
4 & RARG & P13631 & Retinoic acid receptor $\gamma$ & 3.753 & 0.4691 \\
5 & SRC & P12931 & Proto-oncogene tyrosine-protein kinase Src & 3.725 & 0.4139 \\
6 & PTN1 & P18031 & Tyrosine-protein phosphatase non-receptor type 1 & 3.725 & 0.4139 \\
7 & NR1I3 & Q14994 & Nuclear receptor subfamily 1 group I member 3 & 3.72 & 0.6201 \\
8 & SHBG & P04278 & Sex hormone-binding globulin & 3.711 & 0.4638 \\
9 & GSTP1 & P09211 & Glutathione S-transferase P & 3.671 & 0.5244 \\
10 & MAPK14 & Q16539 & Mitogen-activated protein kinase 14 & 3.657 & 0.3657 \\
11 & NR3C1 & P04150 & Glucocorticoid receptor & 3.637 & 0.3306 \\
12 & KIT & P10721 & Mast/stem cell growth factor receptor & 3.628 & 0.4031 \\
\hline
\end{tabular}


Table 1. Cont.

\begin{tabular}{cccccc}
\hline No. & Target Gene & Uniprot ID & Protein Name & Fit & Norm Fit \\
\hline 13 & TTPA & P49638 & Alpha-tocopherol transfer protein & 3.591 & 0.2993 \\
14 & PLA2G2A & P14555 & Phospholipase A2, membrane associated & 3.586 & 0.4482 \\
15 & PPARG & P37231 & Peroxisome proliferator-activated receptor $\gamma$ & 3.549 & 0.507 \\
16 & VDR & P11473 & Vitamin D3 receptor & 3.51 & 0.27 \\
\hline
\end{tabular}

\subsection{Network Construction and Analysis}

Bioinformatics enrichment tools have contributed greatly in the study of gene function analysis in traditional Chinese medicine [14]. To address the challenges of the functional analysis of gene lists, some preliminary studies on the high-throughput enrichment tools were developed. The fished target proteins were further used to map them to diabetes using the PharmGKB, KEGG, CTD and TTD databases and extracted for analysis. The ClueGo analysis for multi-targets of carainterol A is shown in Figure 2.



Figure 2. Functionally grouped network for the candidate target of carainterol A.

The main molecular functions were divided into five categories: insulin receptor substrate binding, insulin receptor binding, protein tyrosine kinase activity, retinoid $\mathrm{X}$ receptor binding and steroid hormone receptor activity. The main pathways included in the ClueGo analysis were as follows: the PI3K-Akt signaling pathway, pathways in cancer, proteoglycans in cancer, the FOXO signaling pathway, the prolactin signaling pathway, the AMPK signaling pathway, the PPAR signaling pathway, the insulin signaling pathway and the thyroid hormone signaling pathway (Figure 3).

In our previous study, carainterol A showed potent glucose consumption activity in $\mathrm{C}_{2} \mathrm{C}_{12}$ muscle cells. Furthermore, it also displayed increasing glucose consumption in a $\mathrm{db} / \mathrm{db}$ mouse model with a micromolar value that is equivalent to that of metformin. The present study indicated that carainterol A possesses multipharmacology attributes through different target groups such as insulin receptor substrate binding, insulin receptor binding and protein tyrosine kinase activity. Thyroid hormone receptor subtype- $\beta$ agonists could improve glucose tolerance and insulin sensitivity in a dose-dependent manner [15]. Therefore, these could be the potential molecular mechanisms by which carainterol A improves glucose utilization. Furthermore, some new activities were found to be 
related with carainterol A, which might offer some new insights for the application of carainterol A. For example, the AKT signaling pathway plays a crucial role in the insulin signaling pathway. Meanwhile, the aberrant AKT signaling is also involved in many sporadic cancers as well as in several dominantly inherited cancer syndromes [16]. Therefore, the probes that have an influence in the AKT signaling might possess antitumor activity. Interestingly, several pathways involved in cancer were highlighted in the ClueGO analysis of carainterol A, such as in prostate cancer.



Figure 3. Pathway grouped network for the candidate targets of carainterol A.

\subsection{Pathway Verification}

Guided by the in silico prediction results, we explored the effects of carainterol A on the insulin signaling pathway. As shown in Figure 4, carainterol A (Y018) showed no impact on the insulin receptor phosphorylation level in HepG2 cells. However, Y018 could significantly increase the protein levels of IRS-1 at $4 \mu \mathrm{mol}$ in a concentration-dependent manner. Moreover, Y018 could increase downstream protein kinase AKT phosphorylation including the S473 and T308 phosphorylation sites.

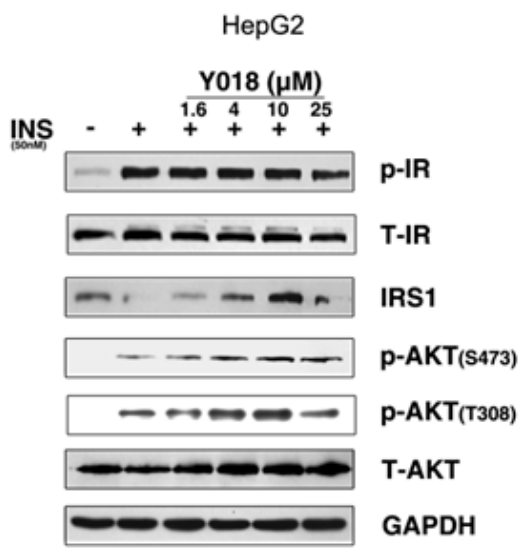

Figure 4. Cont. 


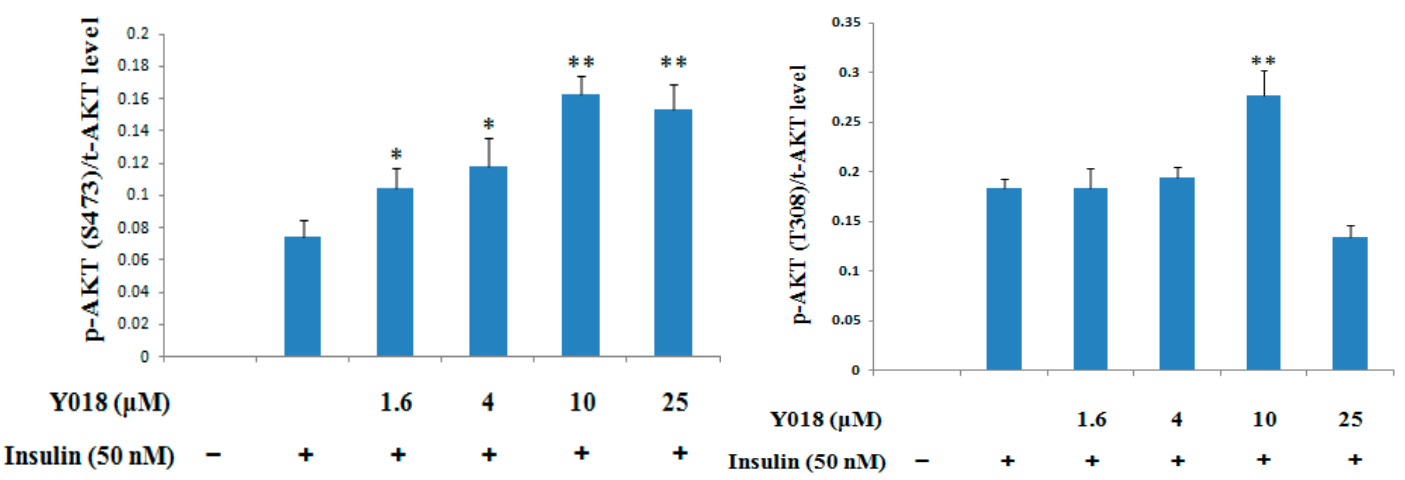

Figure 4. The effect of carainterol $\mathrm{A}$ on the insulin signaling pathway in HepG2 cells. Data are presented as means $\pm \mathrm{SD}\left({ }^{*} p<0.05,{ }^{* *} p<0.01\right)$ from three independent experiments. "+": with insulin; "-": without insulin.

IRS-1 has been implicated in insulin signaling transduction from the insulin receptor to AKT phosphorylation, followed by GLUT4 translocation [17]. To further explore the molecular mechanism of carainterol A, GLUT4 translocation was evaluated to determine whether carainterol A affected the downstream signal. The results indicated that Y018 unexpectedly did not increase the insulin-stimulated human GLUT4 on the membrane at the concentration tested, which was $10 \mu \mathrm{M}$, while the positive compound C2, a known PTP1B selective inhibitor [18], could significantly increase the amount of GLUT4 on the membrane (Figure 5).

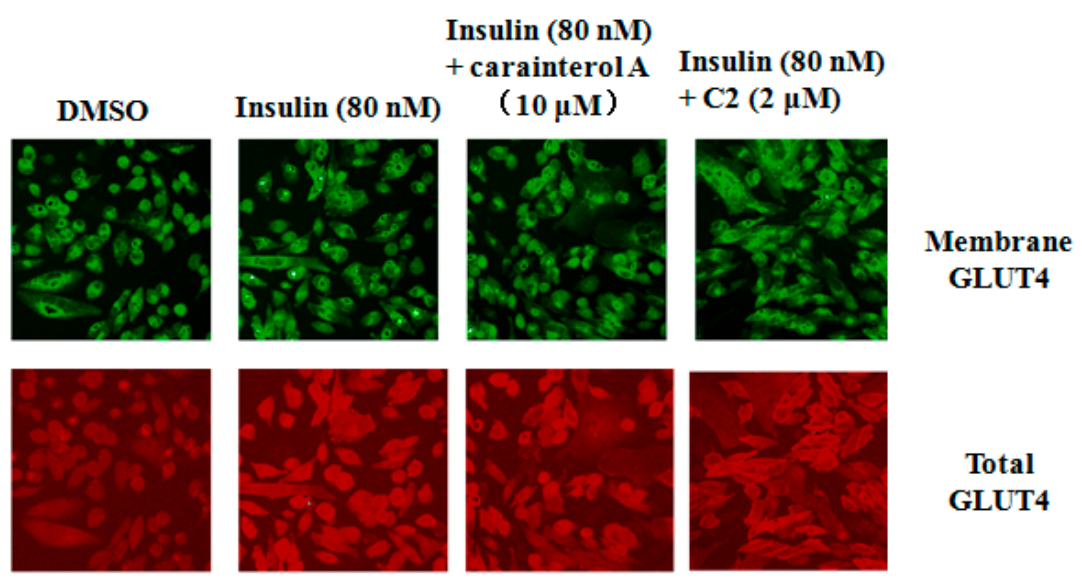

Figure 5. GLUT4 membrane translocation.

\section{Discussion}

Elucidation of the pharmacological profile of a single natural product from natural medicine has been the task for many researchers over the decades. Compared to structural optimization for inhibiting a specific target, multi-target drug approaches were employed recently to study the multi-target character of a single natural product [19]. Among these approaches, network pharmacology was utilized extensively to provide a systemic understanding of the molecular mechanism of natural products. In the current study, the PharmMapper server with pharmacophore reverse screening technology was employed to investigate and predict the potential anti-diabetic targets of carainterol A. The pathway enrichment was analyzed using ClueGO software and the results suggested that carainterol A might have an influence on the insulin signaling pathway and the cancer-related pathway. Eventually, the insulin signaling pathway was selected for experimental validation in order to explore the useful clues for the further study of the molecular mechanism of action. The results indicated that carainterol A could significantly increase the protein levels of IRS- 1 at $4 \mu \mathrm{mol}$. 
The dysregulation of IRS-1 has been implicated in the pathogenesis of type II diabetes and cancer [20]. Thus, it was proposed that therapeutic interventions to mediate the IRS-1 protein level might be a novel approach for the treatment of insulin resistance [21,22]. Recently, it was reported that delayed IRS-1 degradation by the TBC1D3 gene could promote insulin signaling [23]. Moreover, total saponins [24] and Chinese herbal medicine $[25,26]$ had been demonstrated to show anti-diabetic activity through up-regulating the protein expression of IRS-1. To the best of our knowledge, carainterol A is the first natural product that has been reported to increase insulin pathway sensitivity based on the regulation of the IRS-1 level.

Although no influence on GLUT4 translocation was observed, it was proposed that carainterol A may increase glucose consumption through the unknown downstream signaling component of IRS-1. Our results have implied that carainterol A could serve as a valuable chemical probe for the insulin signaling pathway investigation.

\section{Materials and Methods}

\subsection{Target Prediction by PharmMapper}

PharmMapper, a web server for potential drug target identification, is based on the use of a pharmacophore mapping approach [27]. The best mapping poses of the query molecule against all the pharmacophore models in PharmTargetDB is automatically found. The top N best-fitted hits with appropriate target annotations and the aligned poses of the respective molecules are listed. The molecular file of carainterol A was downloaded from the PubChem database (CID: 1548943) and uploaded to the PharmMapper server. The search started using the maximum generated conformations at 300 by selecting "all targets (7302)" option and default value of 300 for the number of reserved matched targets. The default settings were used for other parameters. Their structures were put into the PharmMapper database [28] for target prediction.

In this study, all targets retrieved from the above were sent to database UniProt [29] for target name standardization, which were further subjected to PharmGkb [30]. Therapeutic target database and the comparative toxicogenomics database were employed to eliminate the noise, errors and overlaps to ensure the quality of target database. Afterward, the fished target proteins were further used to map to diabetes using the PharmGKB, KEGG, CTD and TTD databases. Then, the potential targets related to diabetes were extracted from the Therapeutic Target (TTD), DrugBank, and PharmGkb databases.

\subsection{Functional and Pathway Analysis by ClueGO}

ClueGO, a Cytoscape plug-in, is a professional software to enhance the biological interpretation and to analyze the functionally grouped terms in the form of networks and graphs [31]. It was used to perform functional and pathway analysis for the targets related to carainterol A. Simple text version of the targets in gene identifiers type was directly uploaded into the ClueGO software (Institute for Genomics and Bioinformatics Graz University of Technology, Graz, Austria). Based on the hypergeometric distribution, enrichment and depletion tests were employed for terms and groups as two-sided (enrichment/depletion) tests. The network type was adopted as a "Medium" network. In order to develop the annotations network, functional groups were visualized in the network using ClueGO which employed the organic layout algorithm.

\subsection{The Experiments of Carainterol a (Y018) in Insulin-Sensitizing}

The protocol of the pathway verification experiment refers to the paper published by Shen et al. [17]. Y018 was synthesized through the method in our previous study [4].

HepG2 cells were incubated in 12-well plates overnight and the starved cells for $4 \mathrm{~h}$ (maintained compound concentration). Then, the cell was stimulated for $5 \mathrm{~min}$ by the insulin and cells were collected for the Western blot experiments. In the experiments of the regulation of protein level of IRS-1, the compounds were tested after incubation in the serum. 


\subsection{The Influence of Y018 on Membrane Translocation of GLUT4}

In the plasmid pEGFPN1-rGLUT4-myc, c-myc epitope sequence was inserted into the GLUT4 protein extracellular region of rat and the C-terminus was coupled with EGFP green fluorescent protein form a fusion protein. The plasmid was stably transfected into CHO-K1 cells. In the experiment, in the case of impermeable membrane, anti-c-myc antibody as well as the red fluorescent secondary antibodies was used for indirect immunostaining. Green fluorescence intensity indicated the total GLUT4 in the cells and red fluorescence intensity indicated the total GLUT4 on the cell membrane.

\section{Conclusions}

We used PharmMapper and ClueGO software to predict the main disease-related targets and pathways of carainterol A. Furthermore, the effect on the insulin signaling pathway was validated as the main biological mechanism of carainterol A through increasing the protein level of IRS-1 and the downstream protein kinase AKT phosphorylation level. Further studies towards how exactly carainterol A regulates the protein level of IRS-1 are in progress and will be reported in due course.

Acknowledgments: We gratefully acknowledge Xu Sheng, Shanghai Institute of Materia Medica, for help with the work of performing the biological assays. This research was supported by the National Natural Science Foundation of China (No. 21402111), the Basic research program of Shanxi Province (No. 2015021039) and the research project supported by the Shanxi Scholarship Council of China (No. 2015-020).

Author Contributions: Y.M., Y.G., J.T. and L.G. performed the target and pathway predictions. K.M. and D.Y. performed the bioassay to verify the target. K.M. and X.Q. wrote and revised the paper.

Conflicts of Interest: The authors declare no conflict of interest.

\section{References}

1. Newman, D.J.; Cragg, G.M. Natural products as sources of new drugs from 1981 to 2014. J. Nat. Prod. 2016, 79, 629-661. [CrossRef] [PubMed]

2. Fraga, B.M. Natural sesquiterpenoids. Nat. Prod. Rep. 2013, 30, 1226-1264. [CrossRef] [PubMed]

3. Sun, Z.; Chen, B.; Zhang, S.; Hu, C. Four new eudesmanes from caragana intermedia and their biological activities. J. Nat. Prod. 2004, 67, 1975-1979. [CrossRef] [PubMed]

4. Ma, K.; Zhang, C.; Liu, M.; Chu, Y.; Zhou, L.; Hu, C.; Ye, D. First total synthesis of (+)-carainterol A. Tetrahedron Lett. 2010, 51, 1870-1872. [CrossRef]

5. Carlson, E.E. Natural products as chemical probes. ACS Chem. Biol. 2010, 5, 639-653. [CrossRef] [PubMed]

6. Kibble, M.; Saarinen, N.; Tang, J.; Wennerberg, K.; Mäkelä, S.; Aittokallio, T. Network pharmacology applications to map the unexplored target space and therapeutic potential of natural products. Nat. Prod. Rep. 2015, 32, 1249-1266. [CrossRef] [PubMed]

7. Li, S.; Fan, T.P.; Jia, W.; Lu, A.; Zhang, W. Network pharmacology in traditional chinese medicine. Evid. Based Complement. Altern. Med. 2014, 2014, 57-58. [CrossRef] [PubMed]

8. Zhang, B.; Lu, C.; Bai, M.; He, X.J.; Tan, Y.; Bian, Y.Q.; Xiao, C.; Zhang, G.; Lu, A.P.; Li, S. Tetramethylpyrazine identified by a network pharmacology approach ameliorates methotrexate-induced oxidative organ injury. J. Ethnopharmacol. 2015, 175, 638-647. [CrossRef] [PubMed]

9. Manivannan, J.; Silambarasan, T.; Kadarkarairaj, R.; Raja, B. Systems pharmacology and molecular docking strategies prioritize natural molecules as cardioprotective agents. RSC Adv. 2015, 5, 77042-77055. [CrossRef]

10. Kibble, M.; Khan, S.A.; Saarinen, N.; Iorio, F.; Saez-Rodriguez, J.; Makela, S.; Aittokallio, T. Transcriptional response networks for elucidating mechanisms of action of multitargeted agents. Drug Discov. Today 2016, 21, 1063-1075. [CrossRef] [PubMed]

11. Zhang, S.; Zhang, Z.Y. PTP1B as a drug target: Recent developments in PTP1B inhibitor discovery. Drug Discov. Today 2007, 12, 373-381. [CrossRef] [PubMed]

12. Barroso, I.; Gurnell, M.; Crowley, V.; Agostini, M.; Schwabe, J.; Soos, M.; Maslen, G.L.; Williams, T.; Lewis, H.; Schafer, A. Dominant negative mutations in human PPAR $\gamma$ associated with severe insulin resistance, diabetes mellitus and hypertension. Nature 1999, 402, 880-883. [PubMed] 
13. Mukherjee, R.; Davies, P.J.; Crombie, D.L.; Bischoff, E.D.; Cesario, R.M.; Jow, L.; Hamann, L.G.; Boehm, M.F.; Mondon, C.E.; Nadzan, A.M. Sensitization of diabetic and obese mice to insulin by retinoid X receptor agonists. Nature 1997, 386, 407-410. [CrossRef] [PubMed]

14. Huang, D.W.; Sherman, B.T.; Lempicki, R.A. Bioinformatics enrichment tools: Paths toward the comprehensive functional analysis of large gene lists. Nucl. Acids Res. 2009, 37, 1-13. [CrossRef] [PubMed]

15. Grover, G.J.; Singh, R.; Barbounis, P.; Rehmark, S.; Malm, J.; Effenic, S.; Khan, A.; Dong, G.; Bryzgalova, G. Anti-obesity, lipid-lowering, and anti-diabetic effects of the selective thyroid receptor beta agonist KB-141. FASEB J. 2007, 21, A457.

16. Toker, A.; Yoeli-Lerner, M. Akt signaling and cancer: Surviving but not moving on. Cancer Res. 2006, 66, 3963-3966. [CrossRef] [PubMed]

17. Zhang, Y.; Zhang, H.; Yao, X.G.; Shen, H.; Chen, J.; Li, C.; Chen, L.; Zheng, M.; Ye, J.; Hu, L.; et al. (+)-Rutamarin as a dual inducer of both GLUT-4 translocation and expression efficiently ameliorates glucose homeostasis in insulin-resistant mice. PLoS ONE 2012, 7, e31811.

18. Wiesmann, C.; Barr, K.J.; Kung, J.; Zhu, J.; Erlanson, D.A.; Shen, W.; Fahr, B.J.; Zhong, M.; Taylor, L.; Randal, M. Allosteric inhibition of protein tyrosine phosphatase 1B. Nat. Struct. Mol. Biol. 2004, 11, 730-737. [CrossRef] [PubMed]

19. Koeberle, A.; Werz, O. Multi-target approach for natural products in inflammation. Drug Discov. Today 2014, 19, 1871-1882. [CrossRef] [PubMed]

20. Dearth, R.K.; Cui, X.; Kim, H.J.; Hadsell, D.L.; Lee, A.V. Oncogenic transformation by the signaling adaptor proteins insulin receptor substrate IRS-1 and IRS-2. Cell Cycle 2007, 6, 705-713. [CrossRef] [PubMed]

21. Xu, X.; Sarikas, A.; Dias-Santagata, D.C.; Dolios, G.; Lafontant, P.J.; Tsai, S.C.; Zhu, W.; Nakajima, H.; Nakajima, H.O.; Field, L.J.; et al. The CUL7 E3 ubiquitin ligase targets insulin receptor substrate 1 for ubiquitin-dependent degradation. Mol. Cell 2008, 30, 403-414. [CrossRef] [PubMed]

22. Song, R.; Peng, W.; Zhang, Y.; Lv, F.; Wu, H.K.; Guo, J.; Cao, Y.; Pi, Y.; Zhang, X.; Jin, L.; et al. Central role of E3 ubiquitin ligase MG53 in insulin resistance and metabolic disorders. Nature 2013, 494, 375-379. [CrossRef] [PubMed]

23. Wainszelbaum, M.J.; Liu, J.; Kong, C.; Srikanth, P.; Samovski, D.; Su, X.; Stahl, P.D. TBC1D3, a hominoid-specific gene, delays IRS-1 degradation and promotes insulin signaling by modulating p70 S6 kinase activity. PLoS ONE 2012, 7, e31225. [CrossRef]

24. Yu, H.; Zheng, L.; Xu, L.; Yin, L.; Lin, Y.; Li, H.; Liu, K.; Peng, J. Potent effects of the total saponins from dioscorea nipponica makino against streptozotocin-induced type 2 diabetes mellitus in rats. Phytother. Res. 2015, 29, 228-240. [CrossRef] [PubMed]

25. Cai, S.; Sun, W.; Fan, Y.; Guo, X.; Xu, G.; Xu, T.; Hou, Y.; Zhao, B.; Feng, X.; Liu, T. Effect of mulberry leaf (Folium mori) on insulin resistance via IRS-1/PI3K/Glut4 signalling pathway in type 2 diabetes mellitus rats. Pharm. Biol. 2016. [CrossRef] [PubMed]

26. Hu, X.; Wang, M.; Bei, W.; Han, Z.; Guo, J. The chinese herbal medicine FTZ attenuates insulin resistance via IRS-1 and PI3K in vitro and in rats with metabolic syndrome. J. Transl. Med. 2014, 12, 47. [CrossRef] [PubMed]

27. Liu, X.; Ouyang, S.; Yu, B.; Liu, Y.; Huang, K.; Gong, J.; Zheng, S.; Li, Z.; Li, H.; Jiang, H. Pharmmapper server: A web server for potential drug target identification using pharmacophore mapping approach. Nucl. Acids Res. 2010, 38, W609-W614. [CrossRef] [PubMed]

28. PharmMapper Database. Available online: http://59.78.96.61/pharmmapper/ (accessed on 17 April 2016).

29. UniProt Database. Available online: http://www.uniprot.org/ (accessed on 18 April 2016).

30. PharmGkb. Available online: http://www.pharmgkb.org/ (accessed on 19 April 2016).

31. Bindea, G.; Mlecnik, B.; Hackl, H.; Charoentong, P.; Tosolini, M.; Kirilovsky, A.; Fridman, W.H.; Pagès, F.; Trajanoski, Z.; Galon, J. Cluego: A cytoscape plug-in to decipher functionally grouped gene ontology and pathway annotation networks. Bioinformatics 2009, 25, 1091-1093. [CrossRef] [PubMed]

Sample Availability: Samples of the compounds are not available from the authors.

(C) 2016 by the authors; licensee MDPI, Basel, Switzerland. This article is an open access article distributed under the terms and conditions of the Creative Commons Attribution (CC-BY) license (http://creativecommons.org/licenses/by/4.0/). 